\title{
Azimuthons: Spatially Modulated Vortex Solitons
}

\author{
Anton S. Desyatnikov, Andrey A. Sukhorukov, and Yuri S. Kivshar \\ Nonlinear Physics Centre, Research School of Physical Sciences and Engineering, Australian National University, \\ Canberra, ACT 0200, Australia
}

(Received 1 June 2005; published 9 November 2005)

\begin{abstract}
We introduce a novel class of spatially localized self-trapped ringlike singular optical beams in nonlinear media, the so-called azimuthons, which appear due to a continuous azimuthal deformation of vortex solitons. We demonstrate that the azimuthons are characterized by two independent integer indices, the topological charge $m$ and the number $N$ of the intensity peaks along the ring. Each soliton family includes azimuthons with negative, positive, and zero angular velocity.
\end{abstract}

PACS numbers: $42.65 . T g$

Recent progress in the experimental study of nonlinear optical effects in bulk dielectric media opens up many novel possibilities in the study of transverse self-trapping of light and the formation of spatial optical solitons [1]. Spatial optical solitons are stationary self-trapped localized modes in homogeneous self-focusing nonlinear media [2], and they may possess transverse energy flow associated with the complicated phase structure. Two familiar examples include radially symmetric vortex solitons $[3,4]$ and rotating soliton clusters [5] created by several interacting fundamental solitons which rotate opposite to the phase gradient [6].

In this Letter, we introduce a novel class of spatially localized self-trapped optical beams in nonlinear media, the so-called azimuthons, which provide an important missing link between the radially symmetric vortices and rotating soliton clusters. We reveal novel physics of nontrivial rotation of self-trapped modulated optical beams and show that the associated angular momentum has two different contributions. The first contribution is due to the internal energy flow; it comes from a nontrivial phase as in the case of the radially symmetric vortex solitons; this reflects the wave nature of the self-trapped beams. The second contribution appears only when the rotating beam is modulated or fragmented, and it has a "particle" origin. While the latter contribution is important for the soliton clusters, the former one dominates for strongly overlapping beams when the "particle identity" in the beam structure is lost. Surprisingly, as we show below, these two contributions can be of opposite signs, giving birth to the nonrotating modulated singular beams described here as stationary azimuthons.

In particular, we find the spatial solitons whose visible rotation can be directed alongside or opposite to the direction of the energy flow. Furthermore, the internal energy flow can be balanced exactly by the "mechanical" rotation, and, in this case, the truly stationary nonrotating states emerge. The term "azimuthons" for these novel selftrapped states reflects their distinctive modulated profile. The intensity of such states is a spatially localized ring modulated azimuthally, and the phase carries a screw-type dislocation; in contrast to the linear vortex phase $\Psi=m \varphi$, the phase of the azimuthon is a staircaselike nonlinear function of the polar angle $\varphi$. In other words, higher-order spatial solitons can be described in terms of azimuthal deformations of the vortex solitons. We analyze different families of such solutions in both Kerr and saturable nonlinear media and demonstrate that the azimuthons are characterized by two independent integer numbers or azimuthal indices: the topological charge $m$ and the number of the intensity peaks $N$. For the soliton clusters [5,7], the number of peaks satisfies the condition $N \geq 4 m$, while the rotating azimuthons with $N \geq 2 \mathrm{~m}$ can exist in saturable media; in particular, we demonstrate truly stationary nonrotating azimuthons with the indices $m=1$ and $N=3$.

We consider the paraxial propagation of light in an isotropic nonlinear medium with an instantaneous response governed by the nonlinear Schrödinger (NLS) equation,

$$
i \frac{\partial \mathcal{E}}{\partial z}+\frac{\partial^{2} \mathcal{E}}{\partial x^{2}}+\frac{\partial^{2} \mathcal{E}}{\partial y^{2}}+F(I) \mathcal{E}=0,
$$

where the local nonlinearity is described by the function $F(I) \geq 0$, and $I=|\mathcal{E}|^{2}$ is the light intensity. Stationary solutions have the generic form $\mathcal{E}=V(x, y) \exp \left(i k_{0} z\right)$, with the complex envelope $V$ and the propagation constant $k_{0}$. However, the solitons were found only [2,3] in the form $V(x, y)=R(r) \exp (\operatorname{im} \varphi)$, where $R$ is the real envelope and $m$ is an integer.

Looking for a more general class of self-trapped beams, we consider solutions allowing self-similar rotation [8,9] and use the cylindrical coordinates rotating with the angular velocity $\omega: \mathcal{E}=V(r, \varphi-\omega z) \exp (i k z)$. The envelope $V$ satisfies the stationary NLS equation:

$$
\frac{\partial^{2} V}{\partial r^{2}}+\frac{1}{r} \frac{\partial V}{\partial r}+\frac{1}{r^{2}} \frac{\partial^{2} V}{\partial \theta^{2}}-i \omega \frac{\partial V}{\partial \theta}-k V+F\left(|V|^{2}\right) V=0,
$$

where $k$ is the propagation constant in the rotating frame $(r, \theta)$, with $\theta=\varphi-\omega z$. For the vortex solitons, the correspondence between the laboratory frame $(r, \varphi)$ and the rotating frame is defined by the relation $k=k_{0}+m \omega$, where the angular velocity $\omega$ is an arbitrary parameter. 
The method of separation of variables is a widely used approach that allows one to find self-similar solutions for both the fundamental and vortex solitons, when the nonlinearly induced potential has a simple form, such as a radially symmetric shape $F\left(R^{2}\right)$. However, this method does not provide solutions for potentials $F\left(|V|^{2}\right)$ that nontrivially depend on both transverse coordinates. Nevertheless, we show that very accurate approximate solutions predicting the existence and unusual properties of novel azimuthally modulated states can be found analytically employing the standard variational method [10] with the ansatz $V(r, \theta)=\tilde{R}(r) \tilde{U}(\theta)$. Below we present the results for two types of nonlinearities that are common in optical materials.

Nonlinear Kerr medium. - The most familiar nonlinear Kerr medium corresponds to the case $F(I)=I$. Substituting our ansatz into the averaged Lagrangian [10], we derive the variational equations:

$$
\begin{gathered}
\frac{d^{2} R}{d \rho^{2}}+\frac{1}{\rho} \frac{d R}{d \rho}-\frac{g^{2}}{\rho^{2}} R-R+R^{3}=0, \\
\frac{d^{2} U}{d \theta^{2}}-\eta U+|U|^{2} U=0 .
\end{gathered}
$$

Here we introduce the rescaled variables $\tilde{R}(r)=$ $\alpha \sqrt{k_{0}} R\left(\sqrt{k_{0}} r\right)$ and $\tilde{U}(\theta)=\beta U(\theta) \exp (i \Omega \theta)$, where $\rho=$ $\sqrt{k_{0}} r$ with the scaling factor $k_{0}$. Parameters $\alpha, \beta, g$, and

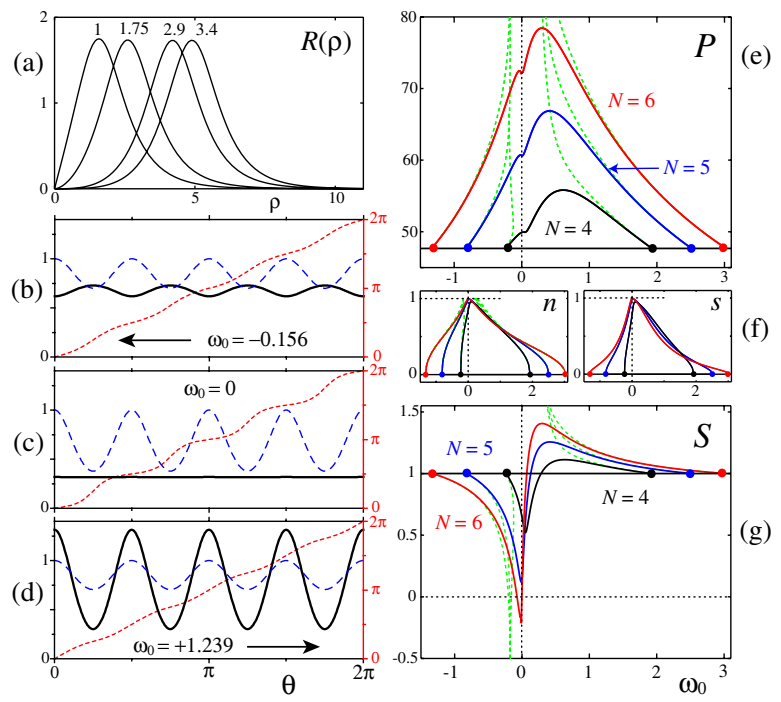

FIG. 1 (color online). (a) Radial envelopes $R(\rho)$ for several values of $g$ shown near the curves. (b) -(d) Azimuthal envelopes $\Phi / A$ (long-dashed line) defined by Eq. (4) and $\Omega \theta+\Psi$ (shortdashed line) defined by Eq. (5) shown for three characteristic cases with $m=+1$ and $N=4$ : (b) $S=0.93$, (c) $S=0.65$, and (d) $S=1.24$. Solid lines in (b) - (d) show the local current along the ring; arrows indicate the rotation direction. (e) Power $P$, (f) parameters $n$ and $s$, and (g) spin $S$ vs the angular velocity $\omega_{0}$, for $m=+1$ and $N=4,5$, and 6. Horizontal solid lines in (e)(g) correspond to the single-charged vortex soliton with $P=$ 48.4, $n=s=0$, and $S=1$. $\eta$ are defined through the integrals over different combinations of the functions $\tilde{R}(r)$ and $\tilde{U}(\theta)$.

We start our analysis with Eq. (2) and find localized solutions for any $g^{2} \geq 1$; several examples are shown in Fig. 1(a). For integer $g=m$, Eq. (2) gives the exact profiles for the vortex solitons. The important parameter is the mean radius of the ring $\rho_{0}$ defined as $\rho_{0}^{-2}=$ $\left[R^{2} \rho^{-2}\right] /\left[R^{2}\right]$, where $[X]=\int_{0}^{\infty} X \rho d \rho$. Parameter $\Omega=$ $\omega_{0} \rho_{0}^{2} / 2$ has the meaning of a mechanical angular momentum of a ring with the mass $1 / 2$ and radius $\rho_{0}$ rotating around its axis with the angular velocity $\omega_{0}=\omega / k_{0}$.

Next we examine the equation for the azimuthal envelope $\tilde{U}(\theta)$ and note that the ringlike solutions should satisfy the condition $\tilde{U}(\theta+2 \pi)=\tilde{U}(\theta) \exp (i 2 \pi m)$ with arbitrary integer $m$. It follows that $U(\theta+2 \pi)=U(\theta) \times$ $\exp [i 2 \pi(m-\Omega)]$, and Eq. (3) is the stationary form of the cubic NLS equation. Corresponding periodic solutions, or cnoidal waves, are characterized by the number of peaks $N$ on the period $\theta \in(0,2 \pi)$. Therefore, the number of peaks $N$ in the intensity distribution of the modulated beam is an additional parameter generally independent on the beam topological charge $m$.

Equation (3) allows one to obtain the structure of the modulated soliton without further approximations. Corresponding solutions have been examined earlier in Ref. [11], and here we generalize these results by taking into account the mode rotation, $\omega \neq 0$. The amplitude and phase of the azimuthal envelope, $U(\theta)=\Phi(\theta) \times$ $\exp [i \Psi(\theta)]$, are given by

$$
\begin{gathered}
\Phi^{2}(\theta)=A^{2}\left[1-n \operatorname{sn}^{2}(\tilde{\theta}, s)\right], \\
\Psi(\theta)=\sqrt{(1-n)(1-s / n)} \Pi(n, \operatorname{am}(\tilde{\theta}, s), s),
\end{gathered}
$$

with $\tilde{\theta}=\theta N K(s) / \pi, \quad A=N K(s) \sqrt{2 s / n} / \pi, \quad$ and two independent parameters: the modulation depth $n$ and the localization parameter $s$. The periodicity condition $\Psi(\theta+$ $2 \pi)=\Psi(\theta)+2 \pi(m-\Omega)$ gives the relation between the parameters $n$ and $s, N^{2} \Pi^{2}(n, s)(1-n)(n-s)=$ $n \pi^{2}(m-\Omega)^{2}$. The notations sn and am stand for the Jacobi elliptic functions, and $K$ and $\Pi$ are the complete elliptic integrals of the first and third kinds, respectively. (a)

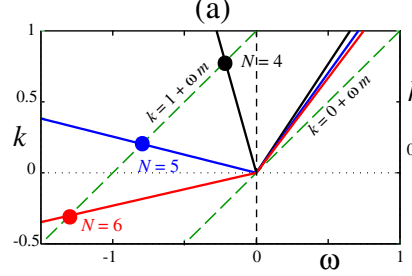

(b)

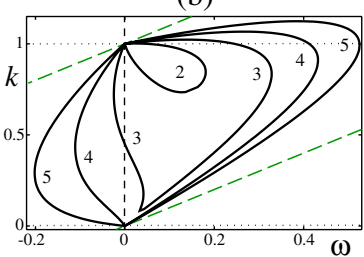

FIG. 2 (color online). Existence domain for the single-charged $(m=+1)$ azimuthons in (a) Kerr and (b) saturable nonlinear media. In (a), the domain is obtained by scaling $\omega=k_{0} \omega_{0}$ and $k=k_{0}+\omega m$; the solid circles correspond to the cutoff points in Figs. 1(e) $-1(\mathrm{~g})$. Solid cutoff lines limit the domain for a soliton with the azimuthal numbers $N$ shown near the curves. 
Solving the variational equations, we obtain the families of the modulated solitons characterized by the azimuthal quantum numbers $m$ and $N$ and parametrized by the angular velocity $\omega$ and the propagation constant $k$; see Figs. 1 and 2(a). These solutions exist in the parameter domain between two cutoff values of the angular velocity, say $\omega^{\text {c.o. }}$, which always have opposite signs. Indeed, we obtain two branches of the solutions in the limit of the vortex soliton, $n \rightarrow 0$, with the cutoff values $\Omega^{\text {c.o. }}=m \pm \sqrt{N^{2} / 4-\rho_{0 m}^{2}}$, where the mean radius of the vortex soliton is $\rho_{0 m}=$ $\lim _{g \rightarrow m} \rho_{0}$, and $\omega_{0}^{\text {c.o. }}=2 \Omega^{\text {c.o. }} \rho_{0 m}^{-2}$. It follows that the azimuthons with the charge $m$ exist for $N \geq 2 \rho_{0 m}$; numerically, $N \geq 4$ for $m=1$, and $N \geq 3 m$ for $m>1$. For negative charges $m \leq-1$, the corresponding curves in Figs. 1(e) $-1(\mathrm{~g})$ should be reflected symmetrically with respect to the line $\omega_{0}=0$, that is, $\omega \rightarrow-\omega$ for $m \rightarrow-m$.

The propagation constant is given by $k=k_{0}+\omega S$; here the spin $S=M / P$ is defined as a ratio of the angular momentum, $M=\mathbf{e}_{z} \operatorname{Im} \int V^{*}(\mathbf{r} \times \nabla V) d \mathbf{r}$, and the beam power, $P=\int|V|^{2} d \mathbf{r}$,

$$
S=m \delta+\Omega(1-\delta),
$$

with the form factor $\delta \equiv 4 \pi^{2}\left\langle\Phi^{2}\right\rangle^{-1}\left\langle\Phi^{-2}\right\rangle^{-1}$, where $\langle X\rangle=\int_{0}^{2 \pi} X d \theta$. Equation (6) demonstrates clearly two different contributions to the angular momentum, the usual term proportional to the dislocation charge $m$, and for the case of a modulated vortex $(\delta<1)$, the mechanical contribution $\sim \Omega$. These two contributions can have opposite signs, if the rotation is opposite to the phase gradient, e.g., $\omega<0$ for $m>0$. In this case, as is seen from Fig. 1(g), the soliton spin can be positive though its value is lower than that of a vortex.

Nonlinear saturable medium. - In general, in nonlinear Kerr media spatial solitons suffer from the collapse instability, and azimuthons make no exception. Therefore, we expand our analysis to the case of saturable nonlinearity, $F(I)=I /(1+I)$ and, because the general ansatz $V=$ $R(r) U(\theta)$ does not lead to integrable equations, we adopt additional simplifications. We consider the limit $s \rightarrow 0$ in Eqs. (4) and (5) and obtain

$$
\begin{gathered}
\Phi_{\operatorname{lin}}^{2}(\theta)=1-n \sin ^{2} \frac{N \theta}{2}, \\
\Psi_{\text {lin }}(\theta)=\frac{2}{N}(m-\Omega) \tan ^{-1} \sqrt{1-n} \tan \frac{N \theta}{2} .
\end{gathered}
$$

Using these expressions, we obtain an algebraic equation for the variational parameter $n$. The radial envelope $R$ satisfies the equation similar to Eq. (2) with the nonlinear potential being the function of $R$ and $n$. The form factor is easy to calculate, $\delta=2 \sqrt{1-n} /(2-n)$, and the parameter $g^{2}=m^{2} \delta+\left(\Omega^{2}+N^{2} / 4\right)(1-\delta)$ has a clear structure with three distinctive contributions. In the "vortex limit," $n \rightarrow 0$ and $\delta \rightarrow 1$, we obtain and solve numerically the equation for the cutoff frequencies.

Figure 2(b) shows the existence domain on the plane $(k, \omega)$ for the azimuthons with $m=+1$. It is limited by the band $\omega m \leq k \leq 1+\omega m$, where vortex solitons exist, $0 \leq$ $k_{0} \leq 1$. In sharp contrast to the Kerr medium with $N \geq 4$, we find bifurcations for the azimuthons with $N \geq 2$. As is clearly seen, a particular solution at $N=2$ can rotate only with a positive velocity $\omega>0$ [8], while the truly stationary azimuthons $\omega=0$ exist for $N \geq 3$. Such a difference with the Kerr model can be explained in terms of the threshold (minimal) power required for generating an azimuthon in the limit $k_{0} \rightarrow 0$. While this is exactly the threshold power of the vortex soliton for $N \geq 4$, there exist additional gaps separating the states with $N=2$ and $N=3$, and bifurcations to these states are not possible in the Kerr media.

Figure 3 shows the characteristic numerical results for the propagation of two azimuthons. For a better comparison, we choose solutions with the same power and modulation depth, both having positive spin but rotating in opposite directions. Equation (6) uncovers a physical origin of this counterintuitive result, namely, two contributions to the angular momentum associated with a dual wave-particle nature of self-trapped nonlinear waves can produce completely opposite effects.

The key issue for understanding an interplay of two effects is the distribution of the energy flow along the ring. Similar to Fig. 1(b) (solid line), the maxima of the

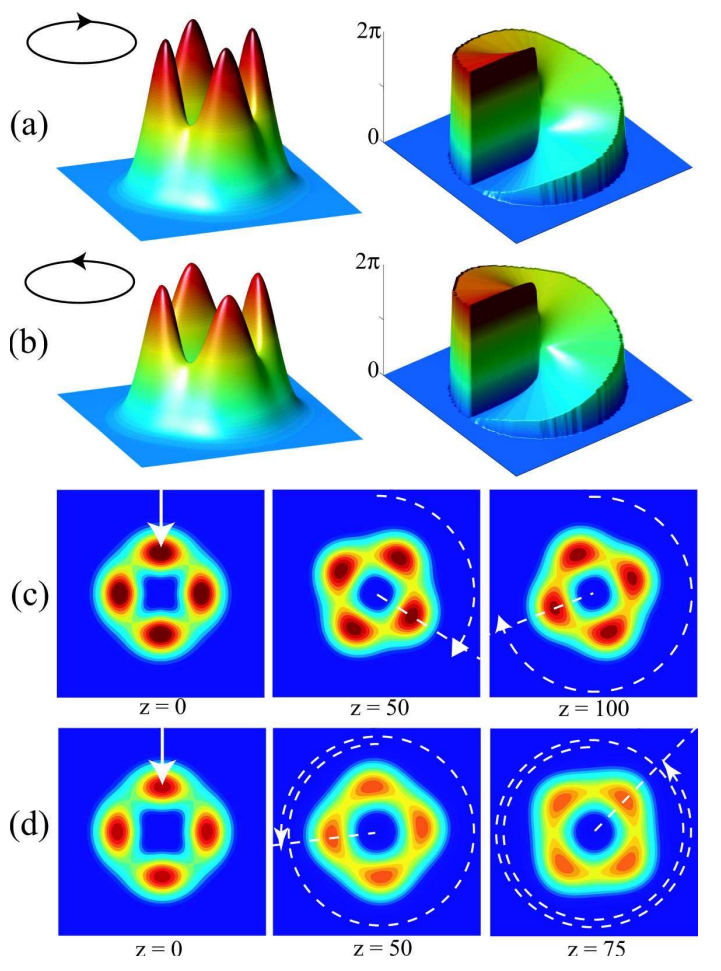

FIG. 3 (color online). Two examples of the azimuthons with $m=1$ and $N=4$. The phase charge $(m=+1)$, modulation depth $(n=0.5)$, and power $(P=440)$ are identical but the predicted angular velocities are opposite: (a),(c) $\omega=-0.087$ with $S=+0.91$ and (b),(d) $\omega=+0.279$ with $S=+1.09$. Numerically averaged angular velocity is (a),(c) $\omega \simeq-0.043$ and (b),(d) $\omega \simeq+0.16$. 


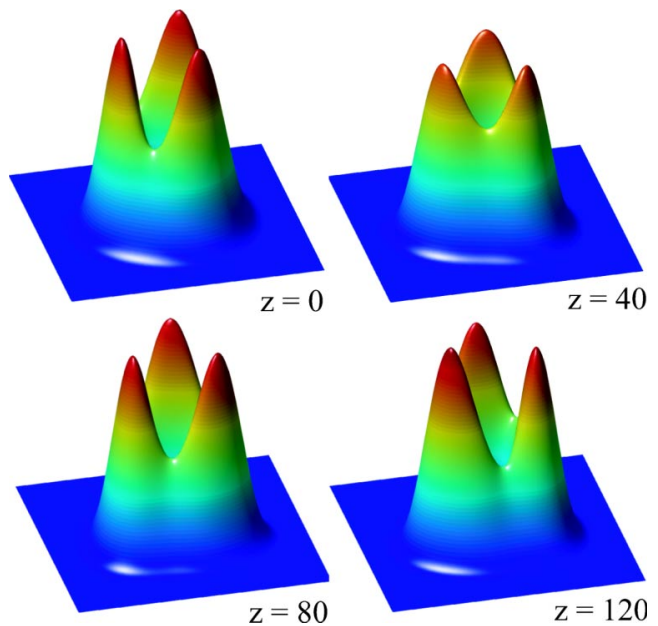

FIG. 4 (color online). Stationary nonrotating azimuthon with $m=1$ and $N=3$ in a saturable medium. Other parameters are $n=0.5, P=2270$, and $S=0.94$. A small twist visible during the propagation is due to weak oscillations.

current along the ring in Figs. 3(a) and 3(c) corresponds to the intensity minima. It means that the regions between the intensity peaks (solitons) transfer the energy faster than the solitons themselves. As a result, the solitons undergo continuous reshaping so that the visible rotation occurs against the energy flow, $\omega<0$. Quite the opposite happens for the solution in Figs. 3(b) and 3(d), analogous to that shown in Fig. 1(d). Here the intensity peaks carry the larger energy and their motion is along the phase gradient; the angular velocity is positive for a positive spin.

Finally, if the energy flow is distributed uniformly along the ring, as in Fig. 1(c), the rotation is suppressed. In Fig. 4 we demonstrate the truly stationary azimuthon with $m=1$ and $N=3$ in a saturable nonlinear medium. We note that, similar to Fig. 3, the dynamics during the propagation is not perfectly stationary because our approximate method does not allow one to obtain exact solutions. However, the azimuthons survive strong oscillations and breathing, so that an additional noise does not affect their dynamics significantly. After the robust propagation for many diffraction lengths, the azimuthons may exhibit symmetrybreaking instability splitting to the fundamental solitons. A linear stability analysis of azimuthons is an interesting open problem.

We underline that the approximations in Eqs. (7) and (8) become unapplicable in the vicinity of the point $\omega=0$; the corresponding (dashed) curves in Figs. 1(e) $-1(\mathrm{~g})$ demonstrate it for the Kerr medium. Thus, the existence of stationary azimuthons with $\omega=0$ and large $N$ remains an open question. Nevertheless, near the cutoff our approximate solutions coincide with the exact solutions for the vortex solitons; thus, the bifurcation diagrams are obtained with a good accuracy. We calculated the existence domain for the azimuthons with the topological charge $m=2$, similar to Fig. 2. Bifurcations to the positively rotating solitons, such as that with $N=2$ in Fig. 2(b), have been found for $N=3,4$, and 5 . For $N \geq 6$, the asymptotics of the cutoff lines in a saturable model originate from the point $(\omega=0, k=0)$, and such double-charged azimuthons are possible in the Kerr model.

In conclusion, we introduced a novel class of spatially localized self-trapped singular beams in self-focusing nonlinear media that provides a missing link between optical vortices and soliton clusters. These singular optical beams, called azimuthons, can be obtained by continuous azimuthal deformation of optical vortices, and they are characterized by two integer indices (or "quantum numbers"), the charge $m$ of the phase dislocation trapped by the beam and the number $N$ of the intensity maxima along the ring. We revealed that the vortex azimuthal deformations are associated with the modulation of the energy flow along the ring resulting in the beam rotation. The rotation can be suppressed by a uniform energy flow along the ring, and then the azimuthon as a whole remains stationary. This provides the first example of multihump nonrotating spatially localized structures in any conservative nonlinear system.

[1] Yu.S. Kivshar and G. P. Agrawal, Optical Solitons: From Fibers to Photonic Crystals (Academic, San Diego, 2003).

[2] R. Y. Chiao, E. Garmire, and C.H. Townes, Phys. Rev. Lett. 13, 479 (1964).

[3] V.I. Kruglov and R. A. Vlasov, Phys. Lett. 111A, 401 (1985).

[4] A.S. Desyatnikov, Yu.S. Kivshar, and L. Torner, in Progress in Optics, edited by E. Wolf (North-Holland, Amsterdam, 2005), Vol. 47.

[5] A.S. Desyatnikov and Yu. S. Kivshar, Phys. Rev. Lett. 88, 053901 (2002).

[6] We note that Fig. 3 of Ref. [5] shows that a soliton cluster rotates opposite to its phase gradient; a similar conclusion holds for the clusters found in all other models [7].

[7] Y. V. Kartashov, G. Molina-Terriza, and L. Torner, J. Opt. Soc. Am. B 19, 2682 (2002); Y. V. Kartashov, L. C. Crasovan, D. Mihalache, and L. Torner, Phys. Rev. Lett. 89, 273902 (2002); A. Desyatnikov and Yu. Kivshar, J. Opt. B 4, S58 (2002); D. Mihalache, D. Mazilu, L. C. Crasovan, B. A. Malomed, F. Lederer, and L. Torner, Phys. Rev. E 68, 046612 (2003); L. C. Crasovan, Y. V. Kartashov, D. Mihalache, L. Torner, Yu. S. Kivshar, and V.M. Perez-Garcia, Phys. Rev. E 67, 046610 (2003); A. Desyatnikov, C. Denz, and Yu. Kivshar, J. Opt. A Pure Appl. Opt. 6, S209 (2004); D. Mihalache, D. Mazilu, L. C. Crasovan, A. Malomed, F. Lederer, and L. Torner, J. Opt. B 6, S333 (2004).

[8] D. V. Skryabin, J. M. McSloy, and W. J. Firth, Phys. Rev. E 66, 055602(R) (2002).

[9] E. G. Abramochkin and V. G. Volostnikov, Phys. Usp. 47, 1177 (2004).

[10] B. A. Malomed, in Progress in Optics, edited by E. Wolf (North-Holland, Amsterdam, 2002), Vol. 43, p. 71.

[11] L. D. Carr, C. W. Clark, and W. P. Reinhardt, Phys. Rev. A 62, 063611 (2000). 